\title{
An Efficient Opportunistic Cooperative Diversity Protocol for IEEE 802.11 Networks
}

\author{
Brett Hagelstein \\ University of Wollongong \\ Northfields Ave, Wollongong \\ NSW 2522, Australia \\ bh02@uow.edu.au \\ Daniel Franklin \\ University of Wollongong \\ Northfields Ave, Wollongong \\ NSW 2522, Australia \\ danielf@uow.edu.au
}

\author{
Mehran Abolhasan \\ University of Wollongong \\ Northfields Ave, Wollongong \\ NSW 2522, Australia \\ mehrana@uow.edu.au \\ Farzad Safaei \\ University of Wollongong \\ Northfields Ave, Wollongong \\ NSW 2522, Australia \\ farzad@uow.edu.au
}

\begin{abstract}
Opportunistic cooperation promises to enhance the user experience when streaming media over wireless devices by improving wireless network reliability at the link level. This paper presents DAFMAC, an efficient cooperative diversity partner selection algorithm for IEEE 802.11 devices. Simulation results show DAFMAC provides a significantly higher transmission reliability in poor channel conditions than traditional ARQ techniques without modifying the device hardware. Further analysis shows the low overhead of DAFMAC makes it highly competitive with other proposed cooperative retransmission mechanisms in an ad-hoc network.
\end{abstract}

\section{Categories and Subject Descriptors}

C.2.2 [Computer Systems Organization]: ComputerCommunication Networks - Network Protocols

\section{General Terms}

Algorithm, Design, Performance, Reliability

\section{Keywords}

MAC protocol, opportunistic cooperative diversity

\section{INTRODUCTION}

Cooperative diversity protocols [6] have been proposed to mitigate the effects of channel fading in wireless environments. The reliability of point to point links is improved by neighbouring devices cooperatively retransmitting lost data packets. This improvement is passed on to the network and application layers, providing a more stable topology and traffic flow, which is particularly important to mobile streaming media.

While a recent testbed implementation by Bradford et al. confirms the predicted diversity gain [3], this work and many others assume an appropriate relay is always available. However, selecting the most appropriate relay from a network is a complex process. Zhao et al. proposed a 'nomination round' for relays to indicate their suitability for cooperation [9]. This selection process scales linearly as devices are added to the network and becomes increasingly inefficient as the size increases. Bletsas et al. eliminates the nomination interaction using a 'method of distributed timers' [2]. This method scales well because relays assume link reciprocity to estimate their ability to participate. Overhead is limited to an RTS/CTS transaction and a relay selection feedback flag.

Other protocols are generally based on these two concepts. $\mathrm{Lu}$ et al. designed and implemented an IEEE 802.11 cooperative MAC layer protocol called PRO [5]. PRO nodes periodically broadcast their link status to nearby nodes to improve the coordination of retransmissions. These broadcasts allow nodes to estimate their cooperative suitability in a distributed fashion to avoid further overhead.

This paper presents DAFMAC - a cooperative decode and forward MAC protocol for IEEE 802.11 devices. It will use existing IEEE 802.11 hardware with a driver modification to coexist with legacy devices. It uses link reciprocity to estimate link quality and a distributed timer scheme to select the best relay. DAFMAC scales well by avoiding inter-relay link status messages.

The contribution of this paper is as follows. The high level relay selection process of DAFMAC described in Section 2. The selection algorithm is developed at the MAC level in Section 3. The simulated performance of DAFMAC is presented in Section 4 with a comparison to 802.11 ARQ behaviour. The cooperative efficiency of DAFMAC is analytically shown to be more efficient than PRO using realistic link estimates in Section 5.

\section{PROTOCOL OVERVIEW}

The basic principles of DAFMAC are as follows. A source node $\mathcal{S}$ transmits a data frame to destination $\mathcal{D}$, where other nodes are available and in communication proximity of both $\mathcal{S}$ and $\mathcal{D}$. This transmission is overheard and decoded by $I$ potential relays, $\mathcal{R}_{i}, i \in[1, I]$, and stored in a buffer. If $\mathcal{D}$ receives the message, it acknowledges with an ACK packet and the relays flush their buffer. Relays expect to hear an ACK after a SIFS period $\left(T_{S I F S}\right)$, as per the IEEE 802.11 MAC standard [4], otherwise they enter a cooperation contention period. The 'best' relay $\mathcal{R}_{b}$ will transmit first and forwards the data frame to $\mathcal{D}$. Other potential relays hear this transmission and return to idle mode.

Relay $\mathcal{R}_{b}$ is the relay with the best channel SNR to $\mathcal{D}$, 
(a) Source Node

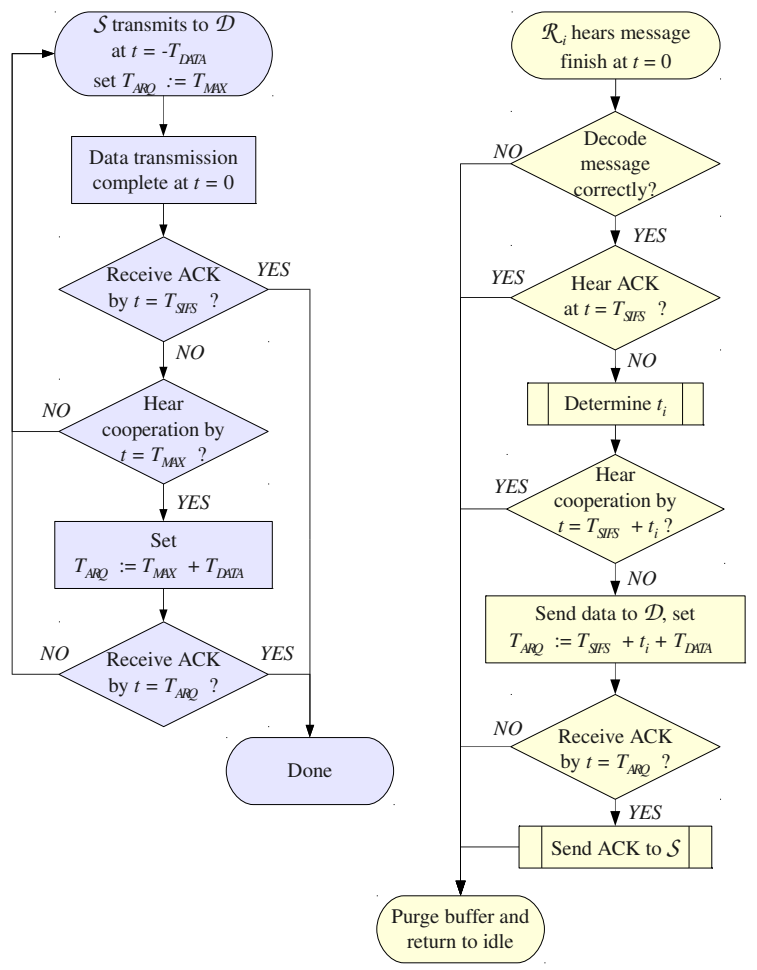

Figure 1: Operation of (a) the source node and (b) potential relay nodes during a cooperative transmission attempt

given by $S N R_{\mathcal{R}_{b} \mathcal{D}} \geq S N R_{\mathcal{R}_{i} \mathcal{D}}$ for $i \in[1, I]$. The channel gain is estimated from the received signal strength (RSS) to $\mathcal{D}$ and the principle of reciprocity. Simulation results show this value is sufficient to select a valid relay even though most 802.11 devices have a low RSS measurement resolution.

The relay selection process uses distributed timers (as used in [2]) where each potential relay will transmit once its timer delay expires. The delay $t_{i}$ allocated to node $\mathcal{R}_{i}$ is inversely proportional to the RSS, hence $\mathcal{R}_{b}$ will have the smallest delay $t_{b} \leq t_{i}$. The algorithm to calculate $t_{i}$ is described in Section 3. All participating nodes reset their timers at the transmission from $\mathcal{S}$

$\mathcal{S}$ retains the traditional ARQ system in the event that there is no suitable relay. However, it extends the time before ARQ is initiated to $T_{A R Q}=T_{S I F S}+T_{M A X}$, where $T_{M A X}$ is the maximum latency of the cooperative algorithm.

$\mathcal{D}$ transmits its ACK to $\mathcal{S}$ via $\mathcal{R}_{b}$ if cooperation was used. There is no acknowledgement of the ACK so $\mathcal{R}_{b}$ assumes the relaying was successful and flushes its buffer when complete. This strategy is validated in Section 4 .

Figure 1a shows the actions and states of $\mathcal{S}$ during both direct and cooperative transmissions, whereas Figure 1b shows the behaviour of all potential relays. $\mathcal{D}$ behaves in the same manner as specified in the 802.11 protocol standard [4].

\section{PROTOCOL DEFINITION}

This section discusses DAFMAC's parameters and how their values are selected.

The IEEE 802.11 MAC standard defines the maximum transmission range $d$ as the distance at which the probability

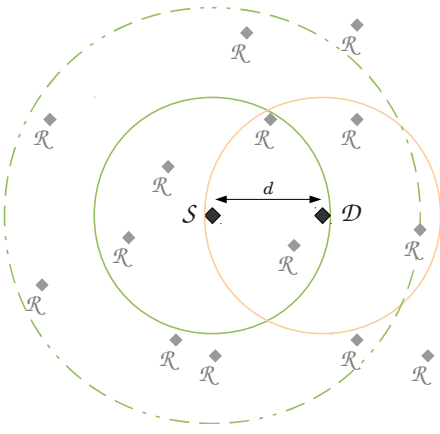

Figure 2: A random layout for $\rho_{\mathcal{R}}=4$; cooperative relays are a subset of nodes and relays are in the interference zone.

of sending a 1000 octet message is $0.9[4] . \mathcal{S}$ and $\mathcal{D}$ are placed $d_{\mathcal{S D}}$ apart where $d$ is used as the unit of distance. The node density, $\rho_{\mathcal{R}}$, is the number of devices with connectivity to $\mathcal{S}$ other than $\mathcal{D}$. This does not indicate the number of nodes with connectivity to both $\mathcal{S}$ and $\mathcal{D}$ as this would require explicit feedback and introduce overhead. Instead, $\rho_{\mathcal{R}}$ can be determined using the 'neighbour table' in each node and this can be used as a probabilistic estimate of the number of participating relays. An example node placement is shown in Figure 2. The expected practical number of devices in connectivity (ie $\rho_{\mathcal{R}}$ ) may reach 20 or more in a dense or long range network.

The delay algorithm uses the following parameters:

- $T_{S L O T}$ - one time-slot as defined by the 802.11 MAC.

- $T_{M A X}$ - the maximum range of delay values that $t_{i}$ can take, measured in units of $T_{S L O T}$.

- $S N R_{\mathcal{S D}}$ - the SNR from $\mathcal{S}$ to $\mathcal{D}$ such that sending 1000 bytes at distance $d$ has a success probability of 0.9 .

- $S N R_{O F F}$ - an offset that determines the minimum link quality of cooperating devices. Relays return to idle unless $S N R_{\mathcal{R}_{i} \mathcal{D}} \geq S N R_{\mathcal{S D}}+S N R_{O F F}$. Values proportional to $\rho_{\mathcal{R}}$ dynamically regulate the number of participating nodes and significantly reduces collisions.

- $S N R_{R N G}$ - the SNR range (in $\mathrm{dB}$ ) expected from relays. If $\mathcal{R}_{i}$ has $S N R_{\mathcal{R}_{i} \mathcal{D}}>S N R_{\mathcal{S D}}+S N R_{O F F}+S N R_{R N G}$ then it has a stronger SNR than expected and will automatically be allocated delay $t_{i}=0$. Otherwise it will have a delay in the range $0 \geq t_{i} \geq T_{M A X}-1$.

Both PRO and the 802.11 ARQ use a minimum i.i.d. delay of 0 to $31 T_{S L O T}$ for collision avoidance. Hence DAFMAC will offer approximately comparable latency and collision avoidance when $T_{M A X}=32$. The timer delay $t_{i}$ is generated from $S N R_{\mathcal{R}_{i} \mathcal{D}}$, as calculated using the RSS and noise measurements already available to the MAC layer. The link budget is the difference in RSS and either receiver sensitivity or ambient noise, although the receiver sensitivity will be the limiting factor in low noise environments. The SNR used is given by

$$
S N R_{\mathcal{R}_{i} \mathcal{D}}=R S S_{\mathcal{D}}-\max \left(S_{R x}, N_{o}\right)
$$

where $S_{R x}$ is the receiver sensitivity and $N_{o}$ is the noise. For example, a RSS of $-62 \mathrm{dBm}$ with a sensitivity of $-88 \mathrm{dBm}$ and noise $-96 \mathrm{dBm}$ would result in a $S N R_{\mathcal{R}_{i} \mathcal{D}}$ of $26 \mathrm{~dB}$.

Relays generate a cooperation rank $C R_{i}$ by

$$
C R_{i}=S N R_{\mathcal{R}_{i} \mathcal{D}}-S N R_{O F F}
$$




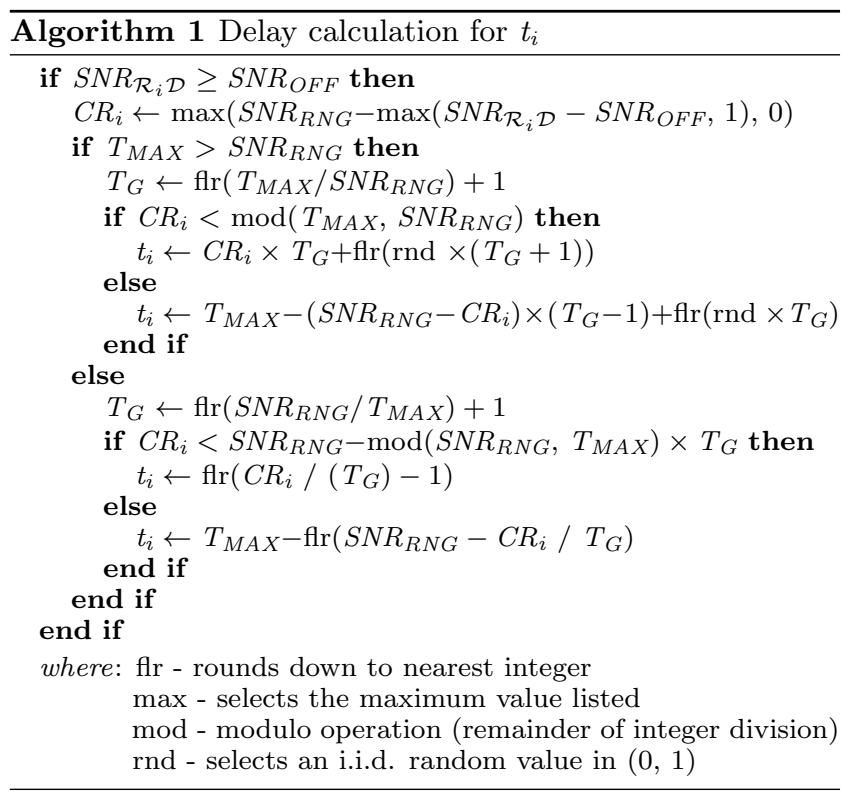

Table 1: Example delay calculation if $S N R_{R N G}<T_{M A X}$

\begin{tabular}{|c|c|c|c|c|c|c|c|c|c|c|}
\hline$C R_{i}$ & \multicolumn{2}{|c|}{1} & \multicolumn{2}{|c|}{2} & \multicolumn{2}{|c|}{3} & 4 & 5 & 6 & 7 \\
\hline$t_{i}$ & 0 & 1 & 2 & 3 & 4 & 5 & 6 & 7 & 8 & 9 \\
\hline
\end{tabular}

Table 2: Example delay calculation if $S N R_{R N G}>T_{M A X}$

\begin{tabular}{|c|c|c|c|c|c|c|c|c|c|c|}
\hline$C R_{i}$ & 1 & 2 & 3 & 4 & 5 & 7 & 9 & 11 & 13 & 15 \\
& & & & & 6 & 8 & 10 & 12 & 14 & 16 \\
\hline$t_{i}$ & 0 & 1 & 2 & 3 & 4 & 5 & 6 & 7 & 8 & 9 \\
\hline
\end{tabular}

Participating relays have $C R_{i} \geq 1$ by definition. Algorithm 1 spreads the range of $C R$ values over period $T_{M A X}$ in a quantised piecewise linear function to create timer delay values that favour higher quality links. This algorithm is computationally efficient and does not require any floating point calculations. Tables 1 and 2 show examples of the delay generation algorithm. The top row indicates the $C R_{i}$ of the relay and the bottom row gives the corresponding delay. A $C R_{i}$ value may be spread over two or more time slots if $T_{M A X}>S N R_{R N G}$, and $t_{i}$ is randomly selected from these values. Conversely, several $C R_{i}$ values may result in the same delay if $S N R_{R N G}>T_{M A X}$.

The RTS/CTS procedure is an option in the IEEE 802.11 $\mathrm{MAC}$ protocol to reduce hidden node interference. $\mathrm{Xu}$ et al. found the RTS/CTS process does not provide significant benefit in ad-hoc environments [8], which is a target application for DAFMAC. Hence DAFMAC can accommodate, but does not require, a RTS/CTS control sequence.

\section{SIMULATION RESULTS}

This section describes the simulation configuration and compares DAFMAC's performance to the ARQ scheme.

\subsection{Network Topology and Configuration}

DAFMAC was simulated using a Monte Carlo method in MATLAB. While not as thorough as a test-bed evaluation, it allowed for rapid evaluation of protocol parameter configurations. The results presented are a probabilistic estimate based on 25000 random network topologies where all devices have an omnidirectional radiation pattern, are co-planar and half-duplex with identical physical layer characteristics.

The parameters used in the simulations were $T_{M A X}=32$, $S N R_{R N G}=64$ and $d_{\mathcal{S D}}=1 d$ unless otherwise specified. These parameter values provided good cooperative performance with a mean timer delay of approximately $15 T_{S L O T}$.

A negative value for $S N R_{O F F}$ results in collisions at high node density $\rho_{\mathcal{R}}$ while a positive value resulted in fewer potential relays for low $\rho_{\mathcal{R}}$. Cooperative success is consistently high when $S N R_{O F F} \propto \rho_{\mathcal{R}}$ as shown in Figure 3. Using $S N R_{O F F}=\rho_{\mathcal{R}}-8$ was experimentally shown to have the best performance for the given parameter set.

\subsection{Results and Discussion}

Cooperation improves the transmission reliability when $S N R_{\mathcal{S D}}$ is reduced, such as when distance $d_{\mathcal{S D}}$ increases due to node mobility. DAFMAC makes a progressively higher contribution to transmission success as node density increases as shown in Figure 4. While the DAFMAC's probability of success is lower than a direct transmission for $d_{\mathcal{S D}}<1 d$, cooperation will rarely be used in this case because of the high reliability of the original transmission. Beyond $d_{\mathcal{S D}}=1.05 d$, DAFMAC improves the reliability by more than $70 \%$ over ARQ for even a low node density. The overall transmission success rate remains high as $d_{\mathcal{S D}}$ increases despite the rapid decline in the reliability of a direct transmission.

The aforementioned parameter values resulted in a mean cooperation latency of about $15 T_{S L O T}$. This is approximately the same retransmission delay as both PRO and ARQ protocols. Hence, for the same per-transmission delay, DAFMAC offers superior performance to ARQ and similar performance to PRO. Protocol overhead is discussed in Section 5 .

DAFMAC provides communication to devices temporarily out of transmission range in a similar fashion to a network layer ad-hoc routing protocol. DAFMAC resides in the link layer of the network stack and uses recent RSS information to estimate an alternate link. Conversely, a routing protocol generally plans the route before transmitting the data. Hence, in the event of an apparent link failure due to a failed direct transmission, the latency of DAFMAC is at least four orders of magnitude lower than the best convergence time of modern proactive routing protocols which may initiate route rediscovery unnecessarily [1]. DAFMAC improves the stability of ad-hoc routing protocols by avoiding intermittent link failures. This is of particular benefit to streaming media where temporary link failures result in severe disruption to the user experience (eg. gaps in audio, frozen or blocky video etc.).

The probability of DAFMAC successfully exploiting cooperation increases with the node density $\rho_{\mathcal{R}}$. However, there are several causes of cooperative failure that limit performance where a 'failure' is an unsuccessful cooperation attempt. The failure modes are: no relays available; relay $\mathcal{R}_{b}$ has poor link quality; two relays collide; and a hidden relay interrupts the transmission. The mean probability of each failure for random topologies is shown in Figure 5. Please note these results are an average of random node placements and the characteristics of each network will differ.

The probability of no relay being available decreases as 


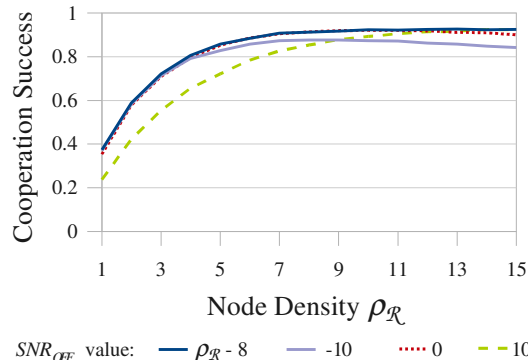

Figure 3: Cooperative success can be consistently high when $S N R_{O F F} \propto \rho_{\mathcal{R}}$

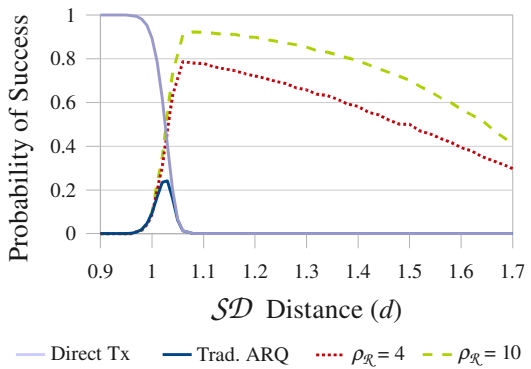

Figure 4: The contribution of DAF$\mathrm{MAC}$ is significantly higher than traditional ARQ

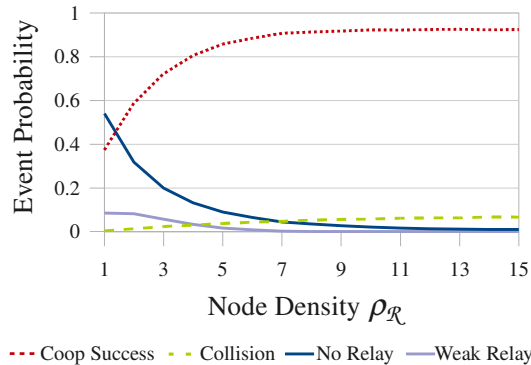

Figure 5: The probability of successful cooperation increases with node density despite the collision increase the node density increases. Decreasing the value of the $S N R_{O F F}$ threshold relaxes the link quality requirement of $\mathcal{R}_{b}$. However, this correspondingly increases the likelihood that $S N R_{\mathcal{R}_{b} \mathcal{D}}$ will be too weak for cooperation. The sum of these failures is approximately constant for a range of $S N R_{O F F}$ values, hence this mode of failure is deemed independent of DAFMAC's performance.

A hidden node failure occurs if a relay begins transmitting after $\mathcal{R}_{b}$ has already started. This will occur when the channel $S N R_{\mathcal{R}_{b} \mathcal{R}_{i}}$ is poor, hence they are hidden from one another. The probability that $\mathcal{R}_{b}$ is close to $\mathcal{D}$ increases with node density $\rho_{\mathcal{R}}$. This reduces the hidden device failure rate because $\mathcal{D}$ is visible to $\mathcal{R}_{i}$ by definition. The probability of hidden nodes is only significant if $S N R_{O F F}$ is negative (ie. very weak links are used as partners) and is negligible under practical operating conditions.

A collision failure occurs when two relays cooperate simultaneously. The collision probability increases with node density as shown in Figure 5. Relaxing the $S N R_{O F F}$ requirement allows more potential relays and increases the chance of collision. A low $S N R_{R N G}$ value increases the chance of two devices having $S N R_{\mathcal{R}_{i} \mathcal{D}}>S N R_{\mathcal{S D}}+S N R_{O F F}+S N R_{R N G}$ and generating a timer delay of 0 . Collisions are reduced using $\rho_{\mathcal{R}}$-dependent value for $S N R_{O F F}$, such as $\rho_{\mathcal{R}}-8$ in this example, because the link quality requirement increases with $\rho_{\mathcal{R}}$ and limits the number of contending relays. Finally, increasing $T_{M A X}$ reduces collisions at the cost of latency.

The relay selection algorithm favours nodes near $\mathcal{D}$ regardless of $S N R_{\mathcal{S R}_{b}}$. Although cooperation will fail if the ACK from $\mathcal{D}$ does not reach $\mathcal{S}$, the simulation showed this was insignificant. If a 1500 byte data frame succeeds with 16- or 64-QAM encoding, a 14 byte ACK using QAM will almost certainly succeed and justifies placing $\mathcal{R}_{b}$ near $\mathcal{D}$.

\section{EFFICIENCY COMPARISON TO PRO}

The PRO MAC protocol developed by Lu et al. operates in a similar manner to DAFMAC except in the relay selection process [5]. Link quality data is periodically shared with other relays, hence PRO relays have greater knowledge of the network topology than DAFMAC relays. Cooperative collisions in PRO are minimised using a probability sum; once the total probability of a successful link surpasses a threshold, additional relays abstain from cooperation as they are more likely to generate collisions than contribute. Hence PRO effectively avoids collisions at the cost of control packet overhead. The comparison question becomes, is the control overhead in PRO less than the collision loss in DAFMAC?
The PRO overhead is calculated as a transmission sum. Each broadcast contains a $T_{D I F S}$ sense period, a (mean) $15 T_{S L O T}$ backoff period, a MAC header and the control data. The data contains the link quality to each node in range and can be compressed into 8 bytes per device. The data length will linearly increase with network density. This process is repeated by all relays within interference range of a node where the interference range is taken as 1.78 times the transmission distance [8]. The Ohd column in Table 3 shows the estimated overhead for networks of varying density using IEEE $802.11 \mathrm{~b}$ at $11 \mathrm{Mb} / \mathrm{s}$ and $802.11 \mathrm{~g}$ at $18 \mathrm{Mb} / \mathrm{s}$ and $54 \mathrm{Mb} / \mathrm{s}$.

PRO is assumed to be ideal, with no collisions but with the same losses as DAFMAC for other failure types. Note the DAFMAC collisions require a further retransmission and some of these may fail as well. This forms a geometric sum and this value is used in the loss calculations. DAFMAC is more efficient if the collision loss is less than the PRO overhead. The number of retransmissions required for the losses to become equal ( $T x$ to Equal in Table 3) is given by:

$$
\text { Tx to Equal }=\frac{\text { PRO overhead }}{\operatorname{Pr}(\text { collision }) \times \text { Tx dur. }}
$$

The duration of transmissions for equal loss (Dur. Tx in Table 3) is estimated by assuming a 1500 byte data packet at the given rate. This total cooperation period is a "per second' value and represents a percentage of the total network utilisation. However, this only includes transmission failures that require cooperative retransmission and can estimate the link quality required for the relative losses to match. The $\operatorname{Pr} . \mathcal{S D}$ Tx column in Table 3 gives the average $\mathcal{S D}$ transmission probability for both protocols to have equal losses. The most efficient example of PRO uses IEEE 802.11g at $54 \mathrm{Mb} / \mathrm{s}$ where the transmission probability is 0.89 . Other configurations can only match the efficiency of DAFMAC at lower levels of link quality.

PRO overhead increases at a higher rate than the DAFMAC loss due to collisions. Hence the $\mathcal{S D}$ channel must become poorer as $\rho_{\mathcal{R}}$ increases if PRO is to achieve efficiency equal to DAFMAC. This behaviour is shown in Figure 6 .

Both protocols are designed for ad-hoc network environments with a network layer routing protocol to ensure link quality. Hence it can be expected that the link reliability will remain above 0.89 and DAFMAC will be more efficient than PRO. Furthermore, PRO will only outperform DAFMAC in highly saturated networks. Wang et al. showed that broadcast control packets become increasingly ineffective for topology control in severely congested networks [7]. 
Table 3: PRO overhead and performance comparison with DAFMAC for different network configurations

\begin{tabular}{|c|c|c|c|c|c|c|c|c|c|c|c|c|c|}
\hline \multirow[b]{2}{*}{$\rho_{\mathcal{R}}$} & \multirow[b]{2}{*}{$\operatorname{Pr}($ coll $)$} & \multicolumn{4}{|c|}{$802.11 \mathrm{~b}(11 \mathrm{M})$} & \multicolumn{4}{|c|}{$802.11 \mathrm{~g}(18 \mathrm{M})$} & \multicolumn{4}{|c|}{$802.11 \mathrm{~g}(54 \mathrm{M})$} \\
\hline & & $\begin{array}{l}\text { Ohd } \\
\text { (ms) }\end{array}$ & $\begin{array}{l}\text { Tx to } \\
\text { Equal }\end{array}$ & $\begin{array}{l}\text { Dur. Tx } \\
\text { (ms) }\end{array}$ & $\begin{array}{c}\text { Pr. } \mathcal{S D} \\
\text { Tx }\end{array}$ & $\begin{array}{l}\text { Ohd } \\
(\mathrm{ms})\end{array}$ & $\begin{array}{l}\text { Tx to } \\
\text { Equal }\end{array}$ & $\begin{array}{l}\text { Dur. Tx } \\
(\mathrm{ms})\end{array}$ & $\begin{array}{c}\text { Pr. } \mathcal{S D} \\
\text { Tx }\end{array}$ & $\begin{array}{l}\text { Ohd } \\
\text { (ms) }\end{array}$ & $\begin{array}{l}\text { Tx to } \\
\text { Equal }\end{array}$ & $\begin{array}{l}\text { Dur. Tx } \\
(\mathrm{ms})\end{array}$ & $\begin{array}{c}\text { Pr. } \mathcal{S D} \\
\text { Tx }\end{array}$ \\
\hline 2 & 0.0130 & 9.24 & 430 & 702 & 0.298 & 2.60 & 230 & 198 & 0.802 & 2.50 & 459 & 190 & 0.810 \\
\hline 6 & 0.0442 & 23.2 & 307 & 501 & 0.499 & 5.60 & 141 & 122 & 0.878 & 5.09 & 272 & 113 & 0.887 \\
\hline 10 & 0.0606 & 41.9 & 398 & 650 & 0.350 & 8.86 & 160 & 138 & 0.862 & 7.79 & 310 & 128 & 0.872 \\
\hline 14 & 0.0664 & 65.2 & 562 & 918 & 0.082 & 12.6 & 207 & 178 & 0.822 & 10.6 & 361 & 149 & 0.851 \\
\hline
\end{tabular}

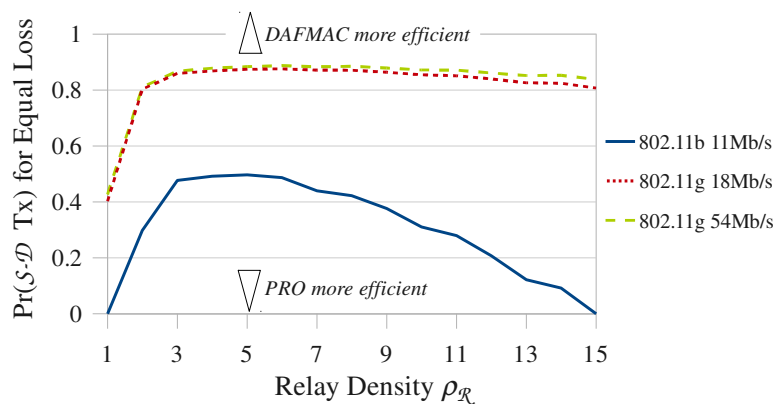

Figure 6: The losses for both protocols will be equal at the $\mathcal{S D}$ transmission probability shown - DAFMAC will be more efficient than PRO if the link quality exceeds this value

Therefore, it can be expected that PRO relay selection will also become unstable and result in a high collision rate as network utilisation increases.

This analysis shows while PRO is an established and effective cooperative MAC protocol, DAFMAC should offer more efficient cooperative retransmission under realistic network assumptions.

\section{CONCLUSION}

This paper presents DAFMAC, a decode and forward MAC protocol that provides opportunistic cooperative diversity for IEEE 802.11 devices without hardware modification.

The protocol, simulated on a simplified model of an IEEE 802.11 device, significantly improves the transmission reliability over a weak channel. These simulations indicate an average improvement in transmission success of more than $70 \%$ over ARQ even with low node density while keeping latency approximately the same. Comparison with PRO's control overhead shows DAFMAC is more efficient under realistic network traffic loads and link quality conditions.

DAFMAC will complement existing ad-hoc routing protocols and reduce packet loss. This will reduce costly route re-discovery at the network layer and has the potential to greatly improve wireless network reliability and provide enhanced performance for streaming media.

The simulated results indicate DAFMAC will provide a solid basis for further research, leading to an implementation on commodity IEEE 802.11 hardware. Further simulations will use QualNet to evaluate network performance when us- ing DAFMAC in conjunction with ad-hoc routing protocols and will focus on providing a consistently high quality link for streaming media and other user applications.

\section{ACKNOWLEDGEMENTS}

This research was partly supported by Australian Research Council grant DP0879507 "Novel Architecture for Next Generation Wireless Relay Networks".

\section{REFERENCES}

[1] M. Abolhasan, B. Hagelstein, and J. C.-P. Wang. Real-world performance of current proactive multi-hop mesh protocols. In Proc. IEEE APCC, pages 44-47, Sep. 2009.

[2] A. Bletsas, A. Lippman, and D. P. Reed. A simple distributed method for relay selection in cooperative diversity wireless networks, based on reciprocity and channel measurements. In Proc. IEEE VTC, volume 3, pages 1484-1488, May 2005.

[3] G. J. Bradford and J. N. Laneman. An experimental framework for the evaluation of cooperation diversity. In Proc. IEEE CISS, pages 641-645, Mar. 2009.

[4] IEEE Computer Society. IEEE Std. 802.11-2007 - Part 11: Wireless LAN Medium Access Control (MAC) and Physical Layer (PHY) Specifications. IEEE Standards Associations, June 2007.

[5] M.-H. Lu, P. Steenkiste, and T. Chen. Design, implementation and evaluation of an efficient opportunistic retransmission protocol. In Proc. ACM MobiCom, pages 73-84, Sep. 2009.

[6] A. Sendonaris, E. Erkip, and B. Aazhang. User cooperation diversity - part I: System description. IEEE Trans. Commun., 51(11):1927-1937, Nov. 2003.

[7] J. C.-P. Wang, M. Abolhasan, D. R. Franklin, and F. Safaei. Characterising the behaviour of IEEE 802.11 broadcast transmissions in ad hoc wireless LANs. In Proc. IEEE ICC, pages 1-5, 2009.

[8] S. Xu and T. Saadawi. Does the IEEE 802.11 MAC protocol work well in multihop wireless adhoc networks? IEEE Commun. Mag., 39:130-137, June 2001.

[9] B. Zhao and M. C. Valenti. Practical relay networks: A generalization of hybrid-ARQ. IEEE J. Sel. Areas Commun., 23(1):7-18, Jan. 2005. 